\title{
Comportamiento alimentario en inmigrantes, aportes desde la evidencia
}

\author{
Food behavior in immigrants, contributions from the evidence
}

\section{RESUMEN}

El objetivo de esta revisión fue describir la evidencia existente respecto del comportamiento alimentario y dieta en inmigrantes, sus características y las dimensiones consideradas para su estudio. Debido al aumento progresivo de la migración de latinoamericanos hacia Chile durante las últimas décadas y la escasa evidencia acerca del posible impacto que genera este nuevo contexto cultural en sus hábitos alimentarios, estado nutricional y salud en general. Se ha reportado que el proceso de migración tiende a provocar un impacto negativo en la salud de los inmigrantes asociados a un mayor riesgo de enfermedades cardiovasculares, diabetes mellitus, malnutrición por exceso y a una peor calidad de vida relacionadas a un mayor grado de asimilación y a conductas alimentarias poco saludables como el consumo de alimentos altamente procesados ricos en grasa y azúcares, mayor frecuencia de comida fuera del hogar y aumento de colaciones dulces. Sin embargo, existen diferencias por sexo, edad, país de origen, nivel socioeconómico, composición del barrio, y tiempo de residencia por lo que resulta necesario generar evidencia del contexto chileno que permita caracterizar el comportamiento alimentario y la dieta de los inmigrantes para diseñar medidas sanitarias culturalmente competentes que favorezcan su salud y calidad de vida.

Palabras clave: Aculturación; Aculturación alimentaria; Comportamiento alimentario; Inmigrantes; Malnutrición por exceso.

\footnotetext{
ABSTRACT

The objective of this review was to describe the existing evidence regarding the dietary behavior and diet of immigrants, their characteristics and the dimensions considered for their study due to the progressive increase in the migration of Latin Americans to Chile during the last decades and the scarce evidence about the possible impact generated by this new cultural context in eating habits, nutritional status and health in general. It has been reported that the migration process tends to have a negative impact on the health of immigrants associated
}

Nelson Hun ${ }^{1,2}$, Alfonso Urzúa ${ }^{1}$.

1. Escuela de Psicología, Universidad Católica del Norte. 2. Escuela de Nutrición y Dietética, Universidad Santo Tomás, Antofagasta.

Dirigir correspondencia a: Nelson Hun Gamboa. Escuela de Psicología, Universidad Católica del Norte. Av. Angamos 0610. Antofagasta, Chile.

Teléfono: (55)2355822.

E-mail: nelsonhunga@santotomas.cl

Este trabajo fue recibido el 07 de junio de 2018. Aceptado con modificaciones: 07 de agosto de 2018. Aceptado para ser publicado: 26 de octubre de 2018.

with an increased risk of cardiovascular diseases, diabetes mellitus, malnutrition due to excess and a lower quality of life related to a greater degree of assimilation and unhealthy behaviors such as the consumption of highly processed foods rich in fat and sugars, greater frequency of eating out and increased sweet snacks. However, there are differences by sex, age, country of origin, socioeconomic level, neighborhood composition and residence time so it is necessary to produce evidence from the Chilean context that allows for the characterization of food behavior and diet of immigrants to design culturally competent measures that favor health and quality of life. Keywords: Acculturation; Eating behavior; Food acculturation; Immigrants; Malnutrition. 


\section{INTRODUCCIÓN}

La migración puede entenderse como el movimiento de personas de un estado a otro o dentro del mismo con independencia de su tamaño, composición o causas, mediante el cual las personas no nacidas en un territorio determinado llegan a él para establecerse'. La Organización de Naciones Unidas (ONU) señala que el número de inmigrantes a nivel mundial alcanzó 244 millones el 2015, proyectándose que esta cifra se duplique a 400 millones para el $2050^{2}$.

Según el Anuario Estadístico Nacional de Migración 2005-2014, en Chile hubo un incremento desde 83 mil migrantes en 1982 a 411 mil migrantes el 2014, lo que corresponde a un 2,3\% de la población total ${ }^{3}$. Al 2017 la población inmigrante residente en Chile equivalía al 4,35\% de la población total. De este total, la distribución de población migrante según país o región de nacimiento en orden decreciente corresponde a: Perú 25,2\%, Colombia $14,1 \%$ y Venezuela $11,1 \%$. Los tres primeros lugares de distribución por regiones en orden decreciente corresponden a la región Metropolitana $65,2 \%$, Antofagasta $8,4 \%$ y Tarapacá $5,9 \%{ }^{4}$.

En este contexto, lógicamente la demanda por servicios a migrantes también ha aumentado, en el caso de las prestaciones en salud han sido beneficiados más de 400 mil extranjeros con independencia de si poseen o no visa definitiva ${ }^{5}$. Algunos de los servicios disponibles son el Examen de Medicina Preventiva (EMP), exámenes, tratamientos, hospitalización, procedimientos quirúrgicos, medicamentos del formulario nacional y atención odontológica ${ }^{6}$. Además de la posibilidad de atención a través del programa AUGE siempre y cuando se cumplan los criterios de acceso ${ }^{6}$.

En el contexto escolar la situación es similar, el número de estudiantes extranjeros matriculados en el sistema entre educación parvularia, básica y media en 2010 alcanzaba las 12.845 matrículas, en 2015 se registraron 17.880, es decir, un 39\% más?.

Esta situación es extremadamente compleja, considerando que es un proceso forzado en donde los migrantes sufren discriminación y exclusión social de los cuales los servicios sanitarios no son la excepción ${ }^{8}$. En este marco, la Organización Mundial de la Salud (OMS) sostiene y enfatiza la necesidad de generar políticas que promuevan el derecho a la salud de la población migrante y que estén a la altura de los desafíos que nos presenta un mundo globalizado. En este contexto propone cuatro necesidades urgentes para abordar esta problemática en la población migrante: (1) Mejores políticas que promuevan el derecho a su salud, (2) Tener en cuenta el idioma y necesidades sanitarias singulares derivadas de este proceso, (3) Un buen sistema de monitoreo de la salud y datos sobre sus necesidades sanitarias y (4) Colaboración entre países y sectores para atender necesidades sanitarias ${ }^{8}$.

Una de las necesidades sanitarias radica en invertir en seguridad alimentaria, entendida no solo como la disponibilidad de alimentos, sino a que estos también deben ser adecuados nutricionalmente para asegurar el correcto desarrollo de las potencialidades humanas y evitar el desarrollo de enfermedades crónicas no transmisibles
(ECNT) y malnutrición ${ }^{9,10}$, debiendo ser culturalmente apropiadas ${ }^{11}$. En este sentido el estudio del comportamiento alimentario (CA) entrega nociones esenciales para la correcta adecuación de la seguridad alimentaria. Por ello el objetivo de esta revisión es describir la evidencia existente respecto al comportamiento alimentario y dieta en población migrante, sus características y dimensiones consideradas para su estudio.

\section{El estudio del comportamiento alimentario (CA)}

Los principales marcos de referencia desde los cuales se ha abordado el complejo fenómeno del estudio del CA son la homeostasis conductual, la ingesta hedónica y el abordaje desde las ciencias sociales y de la conducta.

EI CA desde la perspectiva de la homeostasis conductual puede definirse como el conjunto de acciones que realiza un individuo para ingerir alimentos, en respuesta a una motivación biológica, psicológica y sociocultural ${ }^{12}$, donde el eje central de regulación es la regulación neuroendocrina a nivel hipotalámico ${ }^{13,14}$.

Los modelos de ingesta hedónica ponen énfasis en el sistema de regulación hedónico, asociado a la activación del sistema neuronal de recompensa a un alimento de alta palatabilidad, es decir, alimentos que independientemente de su calidad nutricional producen una sensación de placer, por ejemplo, a través de sus características organolépticas, las cuales son centrales en la elección de un alimento o preparación por sobre otras ${ }^{15}$.

Desde las ciencias sociales y de la conducta, las principales disciplinas de las que deriva el conocimiento sobre comportamiento y alimentación son la antropología, la cual concibe la alimentación como una práctica cultural, abordando tradiciones culinarias enraizadas en la cocina ${ }^{16,17,18,19}$, la sociología, que intenta abarcar todo el ciclo del sistema alimentario, desde su producción ${ }^{20,21,22}$, transitando por el consumo ${ }^{23}$, hasta las significaciones que atribuyen los individuos a los alimentos ${ }^{24,25}$ y la psicología.

En el campo de la psicología desde la década del sesenta comenzaron a desarrollarse algunos modelos explicativos sobre el comportamiento alimentario. En primer lugar, está la hipótesis de la ingesta emocional que sostiene que los estados emocionales y de ánimo pueden influir en el comportamiento alimentario, y a su vez la alimentación puede modificar las emociones y estados de ánimo ${ }^{26,27,28}$. Por otra parte, existe una hipótesis que relaciona la motivación del comportamiento alimentario con atributos externos de los alimentos, como sus características organolépticas y deseabilidad que se potencia con la sensación de hambre y saciedad $^{29}$. Finalmente existe un tercer modelo explicativo denominado teoría de la restricción que sostiene que una vez que un individuo decide restringir su alimentación con la finalidad de disminuir peso, existirá un proceso de contra regulación en donde el individuo romperá su dieta y comerá en abundancia ${ }^{30,31,32}$.

Desde un enfoque más social, otra línea de abordaje del CA ha sido considerarlo como un fenómeno multidimensional 
que se complejiza aún más cuando se cruzan dos culturas, siendo fundamental entender como este proceso de encuentro entre estilos de alimentación impacta en la salud de los inmigrantes. En este marco, cobra especial relevancia el proceso de entrar en contacto con la nueva cultura, donde el migrante comienza una modificación de su repertorio conductual conocido como aculturación psicológica ${ }^{33}$ y que se concreta en la práctica a través del desarrollo de estrategias de aculturación, regidas por el deseo entre mantener la idiosincrasia cultural y el interés de participación de la nueva cultura ${ }^{34}$.

\section{Características del CA en población migrante $y$ factores vinculados}

La evidencia existente sobre el CA y dieta en inmigrantes en Latinoamérica es escasa, lo más cercano al contexto latino es el estudio de migrantes hispanoparlantes en Estados Unidos. Entre los principales factores reportados por la literatura vinculados al CA están los de tipo sociodemográficos, estrategias de aculturación y patrones alimentarios en inmigrantes, maternidad e infancia, apoyo socio-emocional y entornos específicos como la escuela.

\section{Sociodemográficos}

Se ha reportado que el contexto donde se desenvuelven los inmigrantes ejerce influencia en las conductas alimentarias de éstos, por ejemplo se ha encontrado que la composición del vecindario tendría una influencia positiva en el CA, donde a mayor cantidad de inmigrantes prevalecería un CA más saludable ${ }^{35}$. El lugar de nacimiento y el tiempo de permanencia en el país de acogida parecieran ser otros factores influyentes: se ha reportado que existen diferencias en las conductas alimentarias entre inmigrantes nacidos en el extranjero con respecto a inmigrantes de segunda generación, dónde estos últimos tienen una mayor probabilidad de desarrollar sobrepeso, dada por diferencias estructurales como una mayor probabilidad de un ingreso familiar bajo, vivir en lugares con mayor densidad de inmigrantes o menor educación materna ${ }^{36}$. Estudios realizados en mexicanos migrantes en Estados Unidos han mostrado que mientras más prolongado es el tiempo de residencia, mayor es el aumento de peso, principalmente por la rápida asimilación de conductas relacionadas con el sobrepeso, como la dieta, el tabaquismo y la inactividad física $^{36}$. Hechos similares también han sido reportados en asiáticos radicados en Europa posterior a la migración, donde cambios en su conducta alimentaria tales como una tendencia al aumento de la densidad energética, consumo de grasas, carbohidratos refinados, carnes y lácteos, fueron asociados a consecuencias negativas para la salud como el riesgo de obesidad, diabetes mellitus tipo 2 y enfermedades cardiovasculares ${ }^{37}$.

El país de origen también influye. No todos los inmigrantes cambian sus patrones alimentarios de la misma forma, existen diferencias significativas en la prevalencia de conductas alimentarias de riesgo entre niños de origen mexicano frente a latinos de América Central y del Sur, donde, por ejemplo, el consumo de tortas (sandwich) resultó ser un efecto protector de obesidad para los niños centro y sudamericanos y no así para los mexicanos. Sin embargo, existen patrones beneficiosos para todos, como el consumir desayuno, lo que resultó ser un factor protector de obesidad $^{42}$. Del mismo modo han sido reportados aspectos sorprendentes como que el $30 \%$ de niños mexicanos de entre 7 y 9 años y el $24 \%$ de entre 2 y 6 años consumen café a diferencia de niños estadounidenses que reportan un consumo de entre un 1 y $6 \%$ respectivamente ${ }^{38}$.

El sexo podría también estar condicionando el CA, existiendo diferencias de conductas al comparar niños y niñas, reportándose que los hombres consumieron significativamente más granos, alimentos proteicos, frutas y más comida rápida que las mujeres, a la vez que ambos aumentaron el consumo de colaciones dulces ${ }^{39}$.

\section{Estrategias de aculturación y patrones alimentarios en inmigrantes}

Una vez que el migrante entra en contacto con la nueva cultura y se desencadena el proceso de aculturación psicológica son las estrategias de aculturación las que concretan este proceso en la práctica. El modelo categórico propuesto por Berry describe cuatro estrategias de aculturación: Separación, dónde existe un rechazo a la nueva cultura y mantención de la cultura de origen. La integración que se caracteriza por la combinación de ambas culturas, donde el inmigrante mantiene su cultura de origen a medida que incorpora prácticas culturales de la nueva. La asimilación está caracterizada por la adopción total de la nueva cultura y pérdida de la cultura de origen y finalmente la marginalización donde se produce rechazo tanto a la cultura de origen como a la nueva ${ }^{34,40}$.

La literatura reciente ha observado que, en algunos casos, una menor asimilación puede proteger contra los resultados adversos para la salud ${ }^{38}$. Sin embargo, existe evidencia que indica que inmigrantes de habla hispana con un menor nivel de asimilación reportaron significativamente mayor preocupación y menor satisfacción con la salud física de sus hijos y tuvieron las tasas más altas de inseguridad alimentaria. Por el contrario, los padres blancos no hispanos tuvieron una tendencia significativamente mayor a informar conductas alimentarias problemáticas que cualquier grupo hispano $^{41}$. Se ha reportado que mujeres hispanas de bajos ingresos con mayor nivel de asimilación poseen un menor índice de alimentación saludable, consumen menos frutas y verduras y realizan menos actividad física en el posparto que aquellas menos asimiladas o biculturales. Esto se tradujo en que por cada punto de aumento en la escala de aculturación hacia la asimilación hubo un aumento de $0,8 \mathrm{~kg}$ en promedio ${ }^{42}$.

Pareciera ser que las prácticas alimentarias postmigración suelen estar asociadas a un peor estado nutricional ${ }^{43,44}$, peor calidad de vida y un peor estado de salud. En este contexto se ha reportado que tener un hijo 
con una estrategia de aculturación preferente de asimilación en comparación con un hijo bicultural (entendido como la combinación entre el CA del país de origen y el CA del país de acogida), se asocia a cambios negativos en el comportamiento alimentario de la madre, disminuyendo en estas la ingesta de vegetales y aumentando el consumo de azúcar, ingesta de bebidas, porcentaje de calorías provenientes de grasas y la frecuencia de comidas fuera del hogar, con independencia de la estrategia de aculturación de estas ${ }^{45}$.

En adultos la evidencia pareciera seguir la misma tendencia que la de los niños, donde un mayor grado de asimilación se relaciona a un impacto negativo en la salud de los inmigrantes estableciendo asociaciones entre un mayor grado de asimilación y el aumento de peso ${ }^{46,47,48}$ menor consumo de frutas y verduras ${ }^{49}$ y comportamientos alimentarios poco saludables como elevado consumo de alimentos procesados y comida rápida ${ }^{50,51}$.

En un análisis más detallado se describe que la ingesta total de energía, proporción de energía provenientes de grasas saturadas y consumo de azúcar en mujeres mexicanas fue menor en comparación con todas las otras subpoblaciones estudiadas: mexicanas nacidas en México (MAMX - del inglés Mexican Americans' born in Mexico), mexicanas nacidas en Estados Unidos (MAUS- del inglés Mexican Americans' born in United States) y blancas no hispanas (NH - White, del inglés non-Hispanic White). También fueron reportadas diferencias en el consumo de refrescos donde fue significativamente más alto el consumo en MAMX y MAUS, el consumo de gaseosas dietéticas fue significativamente mayor en MAUS y $\mathrm{NH}$ - White y las mexicanas consumieron más leche alta en grasa que los otros subgrupos ${ }^{38}$. Por lo que se reportaron efectos positivos y negativos en distintas categorías de aculturación según grupos de alimentos.

\section{Maternidad e infancia}

Existe evidencia sobre diferencias en preferencias alimentarias donde las madres inmigrantes prefieren la comida tradicional de su país de origen a diferencia de sus hijos inclinados hacia la alimentación de la nueva cultura ${ }^{52}$. Pareciera ser que el efecto de las preferencias alimentarias de los menores sobre los patrones de alimentación es un factor importante, principalmente en lo relativo al consumo de alimentos poco saludables altos en azúcares y grasas ${ }^{44,53}$, al aumento en la densidad energética proveniente de grasas y a un menor consumo de granos relacionadas al estrés ${ }^{54}$. A su vez el menor consumo de frutas y verduras en niños está asociado a una mayor asimilación y mayor inseguridad alimentaria ${ }^{43,55}$.

Se ha encontrado que el estilo parental pudiese también influir en el CA, donde un estilo de alimentación más directivo de madres con bajos ingresos generaría mayor presión en sus hijos para que coman mayores cantidades de alimentos, a la vez que utilizarían alimentos como método de recompensa ${ }^{56}$.

\section{Apoyo socio-emocional}

En el caso de las mujeres sin incluir la maternidad la evidencia indica que los estilos de vida ocupados, la falta de relaciones sociales, la sensación de soledad y el alto nivel de estrés son asociados al desarrollo de enfermedades crónicas y el riesgo se acentúa junto con el tiempo de inmigración ${ }^{49,57}$.

Por otra parte el aislamiento social y la sensación de soledad también influyen en CA poco saludables ${ }^{49}$ reportándose de igual forma mayor consumo de comida rápida fuera del hogar ${ }^{50,51}$. Este contexto propicia la inseguridad alimentaria, relacionada a la baja disponibilidad de ciertos alimentos e ingredientes tradicionales como algunos tipos de verduras o especias y que del mismo modo han sido reportados como factores de riesgo a favor de la elección de productos no saludables ${ }^{44,55}$. En este sentido la dieta poco saludable de alta densidad energética y bajo costo para prevenir el hambre y el estrés relacionados a la inseguridad alimentaria propician el desarrollo de ECV, diabetes o depresión ${ }^{58}$.

\section{Entornos específicos: la escuela}

Los entornos sin duda juegan un rol importante en el desarrollo de la cultura y prácticas alimentarias. En este contexto la inmigración supone un choque de culturas que puede o no modificar el comportamiento y hábito alimentario de los inmigrantes. En este sentido instituciones educativas como las escuelas pueden influenciar positiva o negativamente los hábitos alimentarios migrantes. Se ha reportado una prevalencia de sobrepeso significativamente mayor en escolares migrantes $(12,7 \%$ ) que los no migrantes $(6,9 \%)$ con una fuerte asociación a variables socioeconómicas y de desarrollo infantil con el estado nutricional ${ }^{59}$. En este sentido la escuela cumple un rol mediador esencial en la disminución de disparidad en salud entre niños migrantes y no migrantes ${ }^{60}$. Sobre todo teniendo en consideración que si bien algunos padres reconocen cierta preocupación sobre la obesidad infantil y adulta en general, las preocupaciones sobre obesidad no son una prioridad ${ }^{61}$. Para modificar esta situación la participación de los cuidadores puede ser fomentada transmitiendo la información a través de los niños en las escuelas, abordando las barreras culturales y evitando enfoques que potencialmente puedan ser estigmatizadores para la entrega de mensajes sanitarios ${ }^{62}$.

\section{DISCUSIÓN}

La inmigración es un fenómeno complejo que se proyecta siga aumentando durante las próximas décadas ${ }^{2}$. Chile no ha sido ajeno a esta tendencia y el número de inmigrantes que recibe ha ido en aumento durante los últimos 30 años $^{3}$. Dentro de los múltiples cambios que genera el proceso migratorio en la salud de las personas y que por recomendación de la OMS deben ser prioritarios ${ }^{8}$ la alimentación tiene un rol central en donde gran parte de la evidencia disponible indica un impacto negativo de los patrones alimentarios y el estado nutricional en población 
migrante ${ }^{42,43,44,48,49,56}$. Sin embargo, y en contraposición a los efectos generalmente negativos del proceso de asimilación, existe evidencia de que la americanización de la dieta por parte de mexicanos en estados unidos no significa una reducción en la calidad de la dieta por lo que la asimilación no sería un factor de riesgo ${ }^{58}$. Por otra parte, se ha reportado un efecto amortiguador de la dieta mediterránea en patrones alimentario de inmigrantes residentes en España ${ }^{63}$ para este último caso resulta necesario recordar que la dieta mediterránea declarada patrimonio inmaterial de la humanidad por la $\mathrm{UNESCO}^{64} \mathrm{es}$ un contexto alimentario muy particular como para poder ser fácilmente generalizable. En este sentido todo indica que las dinámicas y características del nuevo contexto alimentario en el que se desenvuelven los inmigrantes son vitales para entender los patrones alimentarios pudiendo haber variantes positivas y negativas en la modificación del CA con el respectivo impacto en su salud y deben ser estudiadas en forma situada.

Dado lo mencionado llama particularmente la atención la escasa evidencia sobre alimentación y nutrición de inmigrantes, situación compleja si se toma en cuenta el aumento de la inmigración en Chile y los reportes de inmigrantes latinos en Estados Unidos, uno de los contextos con evidencia más próximos al nuestro, donde existe una repetida asociación entre asimilación y aumento de peso tanto en niños ${ }^{38,39}$ como en adultos $^{36,37}$. La complejidad es aún mayor si se tiene en cuenta que los patrones alimentarios varían según nivel de aculturación y edad incluso dentro de un mismo grupo familiar ${ }^{45}$, en diferentes grupos de inmigrantes según país de origen y tradiciones alimentarias ${ }^{56}$ y respecto al tiempo de residencia en el nuevo territorio en donde el llamado efecto migrante sano que se produce durante el primer año de llegada desaparece y aumentan los factores de riesgo en el aumento de peso y la salud mental ${ }^{48,65}$. Todo lo mencionado anteriormente se asocia al desarrollo de enfermedades crónicas como ECV, DM II, HTA entre otras ${ }^{49}$. En este contexto Chile no cuenta con referencias propias que permitan abordar los problemas alimentarios y patologías asociadas a estos, por lo que el estudio del CA alimentario en población migrante vinculado al desarrollo de intervenciones educativas culturalmente aceptables en escuelas puede ser una importante estrategia preventiva ${ }^{60,66,67}$.

\section{CONCLUSIÓN}

Resulta urgente generar evidencia que permita caracterizar el comportamiento alimentario, patrones de alimentación y preferencias alimentarias para poder diseñar medidas sanitarias culturalmente competentes que favorezcan la salud y calidad de vida de los inmigrantes durante todo el ciclo vital.

Esta publicación es parte del proyecto FONDECYT \# 1180315, financiado por CONICYT, quienes no tuvieron injerencia alguna en la redacción del texto.

\section{BIBLIOGRAFÍA}

1. International Organization for Migration. Migration glossary [Internet] 2006 [Consulted 2018 may 1]. http://publications. iom.int/system/files/pdf/iml_7_sp.pdf

2. United Nation. OIM projects 400 million migrants by 2050 [Internet]. United Nation; 2010 https://news.un.org/es/ story/2010/11/1205751

3. Ministry of the Interior and Public Security. Migration in Chile 2005-2014 [Internet]. Santiago de Chile: Ministry of the Interior and Public Security; 2016 http://www.extranjeria.gob. cl/media/2016/02/Anuario-Estad\%C3\%ADstico-NacionalMigraci\%C3\%B3n-en-Chile-2005-2014.pdf

4. National Statistics Institute. Censo 2017. http://www.censo2017. cl/descargas/home/sintesis-de-resultados-censo2017.pdf

5. National health fund. Migrant reality in Chile 2016 https:// www.fonasa.cl/sites/fonasa/noticias/2016/07/01/realidad_ migrante_en_chile.html

6. National health fund. Migrant reality in Chile 2017 https:// www.fonasa.cl/sites/fonasa/beneficiarios/extranjeros/preguntasplan\#que-tipo-de-servicios-de-salud-puedo-obtener-comobeneficiario-de-fonasa-

7. Ministry of Education. Undersecretary Quiroga: "One of the challenges of the Reform is to constitute a truly inclusive education system" 2016 https://www.mineduc.cl/2016/09/15/ subsecretaria-quiroga-uno-los-desafios-la-reforma-constituirsistema-educativo-realmente-inclusivo/

8. World Healt Organization. The relevance and importance of promoting health in national SDG responses, Keynote address at the 9th Global conference on health promotion [Internet]. World Health Organization; 29 diciembre 2017 http://www. who.int/dg/speeches/2017/promoting-migrant-health/es/

9. Food and Agriculture Organization of the United Nation. World Food Day [Internet]. Food and Agriculture Organization of the United Nation http://www.fao.org/world-food-day/2017/ theme/es/

10. Pan American Health Organization. Food safety [Internet]. Santiago de Chile. Pan American Health Organization. http:// www.paho.org/chi/index.php?option=Com_content\&view= article\&id=190:seguridad-alimentaria\&/temid=1005

11. Health and human rights. World Health Organization. 2018 http://www.who.int/es/news-room/fact-sheets/detail/humanrights-and-health

12. Oda-Montecinos C, Saldaña C, Beyle C, Andrés A, MoyaVergara R, Véliz-García O. Body dissatisfaction and abnormal eating behaviors in a community sample of Chilean adults. Rev. Mex. de Trastor. Aliment 2018; 9(1): 57-70.

13. Farias G, Netto BDM, Bettini SC, Damaso AR, de Freitas ACT. Neuroendocrine regulation of energy balance: Implications on the development and surgical treatment of obesity. Nutr Health 2017: 23(3): 131-146.

14. Banerjee $S$, Chatuverdi CM. Neuroendocrine mechanism of food intake and energy regulation in Japanese quail under differential simulated photoperiodic conditions: linvolvement of hypothalamic neuropeptides AMPK, insulin and adiponectin receptors. J Photochem Photobiol B. 2018: 185: 10-23.

15. Hernández Ruiz de Eguilaz M, Martínez de Morentin Aldabe B, Almiron-Roig E, Pérez-Diez S, San Cristóbal Blanco R, Navas-Carretero $S$ et al. Multisensory influence on eating behavior: Hedonic consumption. Endocrinol. Diabetes Nutr 2018; 65(2): 114-125.

16. Fischler C. El (H) Omnivore: The taste, the kitchen and the body. Barcelona. Anagrama 1995.

17. Carrasco Henríquez N. Developments in the anthropology 
of food in Latin America: towards the study of contemporary food problems. Social studies 2007; 16(30): 79-101.

18. de Garine V. Anthropology of food. 1ª. Ed. Guadalajara: Universidad de Guadalajara; 2016.

19. Harris M. Good to eat. $2^{a}$. Ed. Madrid; Alianza Editorial; 1989.

20. Montero Mórtola C. The global table. Inter-ethnic eating styles. Gazeta de Antropología 2010; 26(1).

21. Gómez-Correa M. Food culture: Continuities and changes in the preference or rejection and in the methods of food conservation of the migrant population from the Canton of San Pablo (Choco) to Medellin. Eleuthera 2016; 15: 79-98.

22. Morton LW, Bitto EA, Oakland M J, Sand M. Accessing food resources: Rural and urban patterns of giving and getting food. Agric Human Values 2007; 25: 107-119.

23. Sanz Porras J. Sociological contributions to the study of human nutrition: a developing scientific: perspective in Spain. Nutr Hosp 2008; 23(6): 531-535.

24. Rotenberg $S$, De Vargas $S$. Food practices and health care: from feeding the child to feeding the family. RBSMI 2004; 4(1): 85-94.

25. Fernández-Armesto F. History of food: Food, cooking and civilization. $3^{\text {a }}$. Ed. Barcelona; Tusquets Editores, S.A. 2009.

26. Herman $C P$, Polivy J. Anxiety, restraint and eating behavior. J Abnorm Psychol 1975; 84: 666-672.

27. Gibson E. Emotional influences on food choice: Sensory, physiological and psychological pathways. Physiol. Behav 2006; 89: 53-61.

28. Saldaña C. Eating Disorders. Madrid: Fundación UniversidadEmpresa; 1994.

29. López Espinoza A, Martínez Moreno A. From hunger to satiety. Guadalajara, Jalisco: Universidad de Guadalajara; 2011.

30. Herman CP, Mack D. Restrained and unrestrained eating. I Pers 1975; 43: 647-660.

31. Mora Giral M, Raich Escursell R. Food restriction and bulimia nervosa: A causal link?. Annals of Psychology 1993; 9(2): 69-84.

32. Cuadro E, Baile J. Binge eating disorder: analysis and treatment. Rev. Mex. de trastor. Aliment 2015; 6: 97-107.

33. Graves T. Psychological Acculturation in a Tri-Ethnic Community. Southwestern I Anthropol 1967; 23(4): 337-350.

34. Berry JW. A Psychology of Immigration. JSI 2001; 57(3): 615631.

35. Zhang D, van Meijgaard J, Shi L, Cole B, Fielding J. Does neighbouhood composition modify the association between acculturation and unhealthy dietary behaviours?. J Epidemiol Community Health 2015: 724-731.

36. Gordon-Larsen P, Mullan K, Ward D, Popkin B. Acculturation and overweight-related behaviors among Hispanic immigrants to the US: The National Longitudinl Study of Adolescent Health. Soc Sci Med 2003; Dec; 57(11):2023-34.

37. Holmboe-Ottesen G, Wandel M. Changes in dietary habits after migration and consequences for health: a focus on South Asians in Europe. Food \& Nutr Res 2012; 56(1): 18891.

38. Batis C, Hernandez-Barrera L, Barquera S, Rivera J, Popkin B. Food Acculturation Drives Dietary Differences among Mexicans, Mexican Americans, and Non-Hispanic Whites. I Nutr 2011; 141(10): 1898-1906.

39. McCrory M, Jaret C, Kim J, Reitzes D. Dietary Patterns among Vietnamese and Hispanic Immigrant Elementary School Children Participating in an After School Program. Nutrients 2017; 9(5): 460.

40. Zlobina A, Basabe N, Páez D. Immigrantsácculturtive strategies: Their psychological meaning. J Soc Psychology 2008; 23(2):
143-150.

41. Gorman K, Kondo Zearley K, Favasuli S. Does Acculturation Matter?: Food Insecurity and Child Problem Behavior Among Low-Income, Working Hispanic Households. Hisp J Behav Sci 2011; 33(2): 152-169.

42. Martin C, Tate D, Schaffner A, Brannen A, Hatley K, Diamond $M$ et al. Acculturation Influences Postpartum Eating, Activity, and Weight Retention in Low-Income Hispanic Women. J Women's Health 2017; 26(12): 1333-1339.

43. Wiley J, Cloutier M, Wakefield D, Hernandez D, Grant A, Beaulieu A et al. Acculturation Determines BMI Percentile and Noncore Food Intake in Hispanic Children. J Nutr 2013; 144(3): 305-310.

44. Buscemi J, Beech B, Relyea G. Predictors of Obesity in Latino Children: Acculturation as a Moderator of the Relationship Between Food Insecurity and Body Mass Index Percentile. I Immigr Minor Health 2009; 13(1): 149-154.

45. Soto S, Arredondo E, Marcus B, Shakya H, Roesch S, Ayala G. Effects of Latino children on their mothers' dietary intake and dietary behaviors: The role of children's acculturation and the mother-child acculturation gap. Soc Sci Med 2017; 191: 125-133.

46. Franzen L, Smith C. Acculturation and environmental change impacts dietary habits among adult Hmong. Appetite 2009; 52(1): 173-183.

47. Hartwell H, Edwards J, Brown L. Acculturation and food habits: lessons to be learned. Br Food J 2011; 113(11): 13931405.

48. Goulão B, Santos $O$, Carmo I. The impact of migration on body weight: a review. Cad Saúde Pública 2015; 31(2): 229245.

49. Popovic-Lipovac A, Strasser B. A Review on Changes in Food Habits Among Immigrant Women and Implications for Health. J Immigr Minor Health 2013; 17(2): 582-590.

50. Greenberg L, Cwikel J, Mirsky J. Cultural correlates of eating attitudes: A comparison between native-born and immigrant university students in Israel. Int I Eat Disord 2006; 40(1): 5158.

51. Skreblin L, sujoldzic A. Acculturation process and its effects on dietary habits, nutritional behavior and body-image in adolecents. Coll. Antropol 2003; 2: 469-477.

52. Lindsay A, Wallington S, Greaney M, Hasselman M, Machado $M$, Mezzavilla R et al. Sociocultural and Environmental Influences on Brazilian Immigrant Mothers' Beliefs and Practices Related to Child Feeding and Weight Status. Matern Child Health J 2016; 21(5): 1085-1094.

53. Almohanna A, Conforti F, Eigel W, Barbeau W. Impact of Dietary Acculturation on the Food Habits, Weight, Blood Pressure, and Fasting Blood Glucose Levels of International College Students. J Am Coll Health 2015; 63(5): 307-314.

54. Tseng M, Fang C. Stress Is Associated with Unfavorable Patterns of Dietary Intake Among Female Chinese Immigrants. Ann Behav Med 2011; 41(3): 324-332.

55. Dave J, Evans A, Saunders R, Watkins K, Pfeiffer K. Associations among Food Insecurity, Acculturation, Demographic Factors, and Fruit and Vegetable Intake at Home in Hispanic Children. J Am Diet Assoc 2009; 109(4): 697-701.

56. Power T, O'Connor T, Orlet Fisher J, Hughes S. Obesity Risk in Children: The Role of Acculturation in the Feeding Practices and Styles of Low-Income Hispanic Families. Child Obe 2015; 11(6): 715-721.

57. Sliwa S, Must A, Peréa F, Economos C. Maternal employment, acculturation, and time spent in food-related behaviors among 
Hispanic mothers in the United States. Evidence from the American Time Use Survey. Appetite 2015; 87: 10-19.

58. Van Hook J, Quiros S, Frisco M, Fikru E. It is Hard to Swim Upstream: Dietary Acculturation Among Mexican-Origin Children. Popul Res Policy Rev 2015; 35(2): 177-196.

59. Zhou Y, von Lengerke $T$, Walter $U$, Dreier M. Migration background and childhood overweigth in the Hannover región in 2010-2014: A population-Based secondary data analysis of school entry examinations. Eur J Pediatr 2018: 177(5): 753-763.

60. Ji Y, Wang Y, Sun L, Zhang Y, Chang C. The migrant paradox in children and the role of schools in reducing health disparities: A cross-sectional study of migrant and native children in Beijing, China. PLos One 2016: 11(7): 1-12.

61. Greves HM, Lozano P, Liu L, Busby K, Cole J, Johnston B. Immigrant families perceptions on walking to school breakfast: A focus group study. Int J Behav Nutr Phys Act 2007: 4: 64.

62. Jenkins-Berger $E$, Jarpe-Ratner $E$, Giorgio $M$, Squillaro $A$, McCord M, Meyer D. Engaging caregivers in school-based obesity prevention iniciatives in a predominantly latino immigrant community: A qualitative study. J Nutr Educ Behav 2017: 49(1): 53-59.

63. Marín-Guerrero A. C., Rodríguez-Artalejo F, Guallar-Castillón P, López-García E, Gutiérrez-Fisac Juan L. British Journal of Nutrition 2015: 113: 343-349.

64. United Nations Educational, Scientific and Cultural Organization (UNESCO). Mediterranean diet: UNESCO. [Internet]. [Consulted 2018 may 15]. Available from: https://ich.unesco.org/es/rl/ la-dieta-mediterranea-00884

65. Wu Z, Schimmele C. The Healthy migrant effect on depression: variation over time? Can Stud Popul 2005; 32(2):271.

66. López-Espinoza A, Martínez-Moreno A, Aguilera-Cervantes V, Salazar-Estrada J, Navarro-Meza M, Reyes-Castillo Z, GarcíaSanchez N, Jiménez-Briceño A. Study and research of feeding behavior: Roots, development and challenges. Rev Mex de Trastor Aliment 2018: 9(1): 107-118.

67. Salinas J, González C, Fretes G, Montenegro E, Vio F. Theoretical and methodological basis for a healthy food educational program in Chilean basic schools. Rev chil nutr 2014: 41(4): 343-350. 